\title{
Domestic Exposure to Fungi and Total Serum IgE Levels in Asthmatic Children
}

\author{
Huey-Jen Jenny Su, ${ }^{1}$ Pei-Chih Wu, ${ }^{1}$ Huan-Yao Lei, ${ }^{2}$ and Jiu-Yao Wang ${ }^{3}$ \\ ${ }^{1}$ Department of Environmental and Occupational Health, Medical College, \\ National Cheng Kung University, 138 Sheng Li Road, 70428 Tainan, Taiwan \\ ${ }^{2}$ Department of Microbiology and Immunology, Medical College, \\ National Cheng Kung University, 138 Sheng Li Road, 70428 Tainan, Taiwan \\ ${ }^{3}$ Department of Pediatrics, Medical College, National Cheng Kung University, 138 Sheng Li Road, 70428 Tainan, Taiwan
}

Received 9 March 2005; accepted 21 March 2005

\begin{abstract}
We measured the number of airborne, viable fungi and house dust mite (HDM) allergen levels in the homes of a group of asthmatic children. Blood samples were drawn and the amounts of total and specific serum IgE were determined. The association between the number of fungal colonies, dust mite allergen exposure, and specific and total IgE was evaluated. The number of viable airborne fungi was high $\left(20543 \mathrm{CFU} / \mathrm{m}^{3}\right)$ in those investigated houses. Der $p 1$ concentrations on child's mattress exceeding $2 \mu \mathrm{g} / \mathrm{g}$ were found in $78.6 \%$ of the houses. A quantitative dose-response relationship was demonstrated between the exposure to viable, airborne molds and the amount of total $\operatorname{IgE}(r=0.4399$ and $P=.0249)$ and the level was further increased in children with coexposure to viable fungi and HDM.
\end{abstract}

\section{INTRODUCTION}

Fungi are part of the normal environment but exposure levels much over normal may occur indoors in buildings with humidity problems, either due to climate or due to local water damage $[1,2]$. Several studies have reported that children living in damp and moldy dwellings have a larger prevalence of respiratory symptoms, such as cough, phlegm, wheeze, sore throat and runny nose, as well as asthma $[3,4]$. There are however only limited data demonstrating dose relationships between mold exposure and the extent of symptoms or clinical markers of inflammation or asthma. In one field study a relation was reported between the extent of upper respiratory symptoms and the exposure to molds, expressed as the amount of airborne $(1 \rightarrow 3)-\beta$-D-glucan [5].

Taiwan is located in a subtropical region with optimal environmental characteristics, such as high temperature and relative humidity that sustain the growth of molds and indoor aeroallergens. Our previous work demonstrated that $65 \%$ of childrens' mattresses in Taiwan's homes had Der $p 1$ allergen levels higher than that $2 \mu \mathrm{g} / \mathrm{g}$, a critical level suggested for atopic sensitization $[6,7]$.

Correspondence and reprint requests to Jiu-yao Wang, Department of Pediatrics, Medical College, National Cheng Kung University, 138 Sheng Li Road, 70428 Tainan, Taiwan; a122@ncku.edu.tw
In this study, we measured the number of airborne, viable fungi and house dust mite (HDM) allergen levels in the homes of a group of asthmatic children. Blood samples were drawn and the amounts of total and specific serum IgE were determined. The association between the number of fungal colonies, dust mite allergen exposure, and specific and total $\operatorname{IgE}$ was evaluated.

\section{MATERIALS AND METHODS}

\section{Study subjects}

A randomly selected subgroup of asthmatic children was chosen from our previous environmental survey of domestic exposures to dust mite allergens and airborne fungi in a metropolitan city of southern Taiwan [7]. A total of 28 subjects, aged 10-12 years, were invited through phone contact for clinical confirmation conducted by participating pediatricians for their asthmatic status and blood samples were drawn. The clinical visits took place in winter (December, January, and February) at the same time as the environmental measurements.

\section{Airborne fungi sampling and identification}

Airborne fungal concentrations were measured in the subject's bedroom once a month during the season. Duplicate samples of airborne fungi were collected using Burkard sampler, a single-stage hole-to-agar impactor with 100 holes (Model PASA/B, Burkard Mfg Co, Rickmansworth, UK) with Malt Extract Agar plates 
TABLE 1. Domestic fungal and Der p1 concentrations and the corresponding serum total IgE levels in 28 asthmatic children.

\begin{tabular}{llllll}
\hline Parameters & Genera & Mean & SD & Medium & 25th-75th quartile \\
\hline & Total fungi & 20543 & 8882 & 19359 & $15350-24525$ \\
& Aspergillus & 328 & 1130 & 78 & $0-122$ \\
Airborne fungi & Penicillium & 445 & 268 & 399 & $284-586$ \\
$\left(\mathrm{CFU} / \mathrm{m}^{3}\right)$ & Cladosporium & 17691 & 8558 & 164 & $12514-21908$ \\
& Alternaria & 188 & 188 & 169 & $0-248$ \\
& Nonsporulating & 713 & 486 & 538 & $360-973$ \\
$\operatorname{Der} p 1(\mu \mathrm{g} / \mathrm{g}$ dust $)$ & Yeast & 387 & 375 & 278 & $156-533$ \\
Serum total IgE $(\mathrm{kU} / \mathrm{L})$ & - & 6.72 & 5.38 & 4.52 & $2.56-11.23$ \\
& - & 772.27 & 627.63 & 591.00 & $277.50-1209.50$ \\
\hline
\end{tabular}

(MEA) at a flow rate of 10 LPM. After field sampling, the plates were incubated at $25^{\circ} \mathrm{C}$ for 5 days.

The yields of colonies were corrected by using positive-hole conversion table before the calculation of final concentrations, colony forming unit per cubic meter $\left(\mathrm{CFU} / \mathrm{m}^{3}\right)$. The average concentrations of monthly measurements (December, January, and February) were used to express the airborne fungal exposure levels.

\section{House dust sampling and Der $p 1$ analysis}

Dust samples from the child's mattress were collected by a hand-held vacuum cleaner (National MC 33000) with a glass fiber filter and collected concurrently along the airborne samples. Samples were collected by vacuuming for 3 minutes on $1 \mathrm{~m}^{2}$ of the study area. After the sampling, the samples were sealed with parafilm and stored at $4^{\circ} \mathrm{C}$ before handling. After weighting, all samples were stored with desiccating agents until extraction. Samples were in BBS-T $(0.1 \%$ Tween 20 in borate buffer saline, $\mathrm{pH}$ 8.0 ) and extracted in the ultrasonic vibrator for 2 hours at $25^{\circ} \mathrm{C}$. Der $p 1$ was measured by two-side, monoclonalantibody-based ELISA [8]. The allergen concentrations were expressed in $\mu g$ of measured allergen per gram of dust and the average concentrations of monthly measurements were used to quantify the Der $p 1$ exposures.

\section{Total serum IgE and specific IgE measurement}

Total serum IgE and specific IgE antibodies to Dermatophagoides pteronyssinus, Cladosporium herbarum, and Aspergillus fumigatus were measured by a quantitative fluoroimmunoassay (CAP-FEIA; Parmacia Up\&John, Sweden, detection limit $0.35 \mathrm{kU} / \mathrm{L}$ for total IgE and specific IgE). The standard curve was examined in triplicate samples and within the range of $10 \%$ of coefficient of variation. Children were considered as sensitized if the $\operatorname{IgE}$ concentrations exceeded $0.35 \mathrm{kU} / \mathrm{L}$.

\section{RESULTS}

The number of viable airborne fungi was high (medium: 19359, 25th-75th quartile: 15350$24525 \mathrm{CFU} / \mathrm{m}^{3}$ ) in those investigated houses. The predominant indoor genera were Cladosporium,
Aspergillus, Penicillium, Alternaria, nonsporulating fungi and yeast. Cladosporium accounted for almost $85 \%$ of the colonies from all indoor samples and was present in high amounts $\left(17691 \mathrm{CFU} / \mathrm{m}^{3}\right)$. Der $p 1$ concentrations on child's mattress exceeding $2 \mu \mathrm{g} / \mathrm{g}$ were found in $78.6 \%$ of the houses. The average concentration of Der $p 1$ was $6.72 \mu \mathrm{g} / \mathrm{g}$ (Table 1). No significant relationship was found between levels of Der $p 1$ and viable fungi $(r=0.13)$.

Among the 28 children, 23 were found to sensitize to HDM. One child was sensitiz to $C$ herbarum allergen, and two to A fumigatus extract; all three of them were also HDM-sensitiz. Total serum IgE levels ranged from 18.8 to $2000.0 \mathrm{kU} / \mathrm{L}$, and the average concentration was $772.3 \mathrm{kU} / \mathrm{L}$.

As illustrated in Figure 1, there was a positive and significant correlation between the number of airborne fungi and the amount of total $\operatorname{IgE}(r=0.4399$ and $P=.0249)$; yet, such a relationship could not be detected between $D e r$ p1 levels and total IgE. Study subjects were grouped by the median of viable fungi and Der $p 1$ concentrations, respectively. Logistic regression identified that the only significant relationship was between the dichotomized level of viable fungi and $\operatorname{IgE}$ with $\mathrm{OR}=5.06$ (95\% CI: 1.02-30.06) in univariant analysis. Moreover, an increased OR of 6.67 (95\% CI: 1.19-55.16) between the exposure and response, and the IgE levels, was found when the exposures to airborne fungi and dust Der $p 1$ were concurrently examined in a multivariate analysis.

\section{DISCUSSION}

Although the sample size was relatively small in this pilot investigation, the sample was selected randomly from the general asthmatic population in the study city. Therefore, these results still may reflect the real phenomenon of asthmatic population in this region.

The major finding in the study was the relation between the number of viable fungi and the total amount of $\mathrm{IgE}$ in serum. Similar findings are reported from a study conducted in a mold-contaminated school building where elevated IgE levels were significantly more common among children in mold-contaminated buildings [9]. Another study was performed on persons living in 


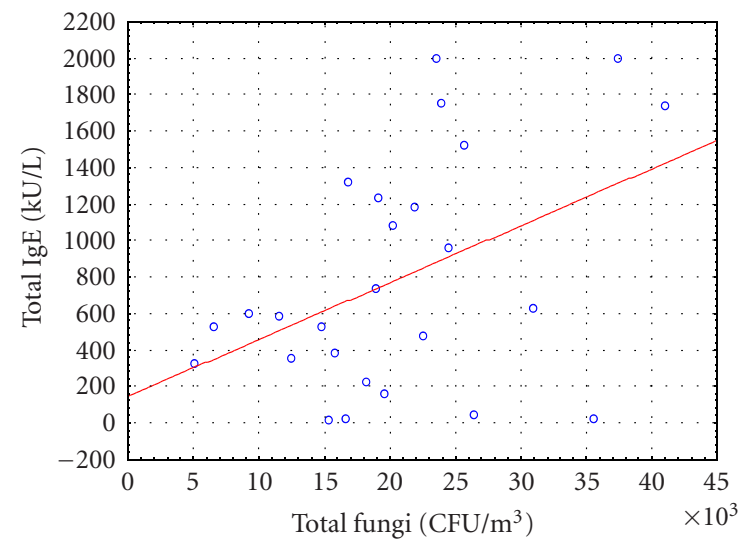

Figure 1. The relationship between total airborne fungal concentrations and serum total IgE levels. $Y=144.9138+0.0312 * \mathrm{X}$, $r^{2}=0.1925 ; r=0.4388, P=.0249$.

houses with mold problems, using the mold cell wall agent $(1 \rightarrow 3)-\beta$-D-glucan as a marker for mold cell biomass. Total IgE levels were dose-related to the amount of $(1 \rightarrow 3)$ $\beta$-D-glucan in the homes, independent of atopic sensitization (Beijer et al, personal communication).

The presence of a relation between mold exposure and total IgE increase and the very low prevalence of sensitization to mold among the children studied suggests that a major effect of mold exposure is a general stimulation of the IgE production without specific sensitization. An increased level of total $\operatorname{IgE}$ on the other hand implies a larger risk for sensitization to other agents, as evidenced by the high prevalence of sensitization to HDM. More than $80 \%$ of the 28 children were sensitized to $D$ pteronyssinus. This rate is similar to that of the $D$ pteronyssinus sensitization pattern reported from UK and other countries $[10,11]$ but higher than that found in school-age children in Taipei, Taiwan (52\%) [12]. It can be hypothesized that the exposure to mold in that group was lower than that in the houses of the children studied here.

Data from previous studies on the effects of inhaled $(1 \rightarrow 3)-\beta$-D-glucan suggest that this agent has the capacity to trigger a $\mathrm{T}_{\mathrm{H}} 2$-like cytokine secretary pattern $[13,14]$. It is also likely that $(1 \rightarrow 3)-\beta$-D-glucan, though not an inflammagenic agent in itself, increases the sensitivity to other inflammagenic agents. If these reaction patterns are correct, a primary reason for the asthmatic status among the children could be the exposure to molds. On the other hand, the home environment contains a variety of other microbial agents such as endotoxin and muramic acid. There are thus several agents in the indoor air environment that could explain the presence of an inflammatory response among the exposed children.

In summary, a dose-response relationship was demonstrated between the exposure to viable, airborne molds and the amount of total IgE and risk was increased as children were under the coexposure to viable fungi and HDM. This suggests that exposure to molds causes an increased sensitivity to other antigens in general and possibly also increases the sensitivity to inflammagenic agents.

\section{ACKNOWLEDGMENTS}

The authors wish to thank Ms Chu-Yun Huang and Ms Fang-Chun Lee for their friendly and laboratory assistance during the study. Most of all, our appreciation goes to all participants and their families for the patience and cooperation. We are eternally in great debt to this support. This study was supported in part by a grant from the Taiwan National Science Council, NSC 86-2621-P006-013.

\section{REFERENCES}

[1] Garrett MH, Rayment PR, Hooper MA, Abramson MJ, Hooper BM. Indoor airborne fungal spores, house dampness and associations with environmental factors and respiratory health in children. Clin Exp Allergy. 1998;28(4):459-467.

[2] Verhoeff AP, van Wijnen JH, Brunekreef B, Fischer P, van Reenen-Hoekstra ES, Samson RA. Presence of viable mould propagules in indoor air in relation to house damp and outdoor air. Allergy. 1992;47(pt 1):83-91.

[3] Hu FB, Persky V, Flay BR, Richardson J. An epidemiological study of asthma prevalence and related factors among young adults. J Asthma. 1997;34(1):6776.

[4] Cuijpers CE, Swaen GM, Wesseling G, Sturmans F, Wouters EF. Adverse effects of the indoor environment on respiratory health in primary school children. Environ Res. 1995;68(1):11-23.

[5] Rylander R, Persson K, Goto H, Yuasa K, Tanaka S. Airborne Beta-1,3-glucan may be related to symptoms in sick buildings. Indoor Environment. 1992;1:263-267.

[6] Su HJ, Wu PC, Chen HL, Lee FC, Lin LL. Exposure assessment of indoor allergens, endotoxin, and airborne fungi for homes in southern Taiwan. Environ Res. 2001;85(2):135-144.

[7] Platts-Mills TA, Vervloet D, Thomas WR, Aalberse $\mathrm{RC}$, Chapman MD. Indoor allergens and asthma: report of the third international workshop. J Allergy Clin Immunol. 1997;100(pt 1):S2-S24.

[8] Luczynska CM, Arruda LK, Platts-Mills TA, Miller JD, Lopez M, Chapman MD. A two-site monoclonal antibody ELISA for the quantification of the major Dermatophagoides spp allergens, Der $\mathrm{p} \mathrm{I}$ and Der $\mathrm{f}$ I. J Immunol Methods. 1989;118(2):227-235.

[9] Savilahti R, Uitti J, Roto P, Laippala P, Husman T. Increased prevalence of atopy among children exposed to mold in a school building. Allergy. 2001;56(2):175-179. 
[10] Pollart SM, Chapman MD, Fiocco GP, Rose G, Platts-Mills TA. Epidemiology of acute asthma: IgE antibodies to common inhalant allergens as a risk factor for emergency room visits. J Allergy Clin Immunol. 1989;83(5):875-882.

[11] Sarsfield JK. Environmental, clinical, and immunological study of house dust mite in childhood asthma. Arch Dis Child. 1973;48(8):655.

[12] Tang RB, Tsai LC, Hwang HM, Hwang B, Wu KG, Hung MW. The prevalence of allergic disease and IgE antibodies to house dust mite in schoolchildren in Taiwan. Clin Exp Allergy. 1990;20(1):33-38.

[13] Rylander R, Fogelmark B. Inflammatory responses by inhalation of endotoxin and $(1 \rightarrow 3)$-beta-Dglucan. Am J Ind Med. 1994;25(1):101-102.

[14] Beijer L, Thorn J, Rylander R. Effects after inhalation of $(1 \rightarrow 3)$-beta-D-glucan and relation to mould exposure in the home. Mediators Inflamm. 2002;11(3):149-153. 


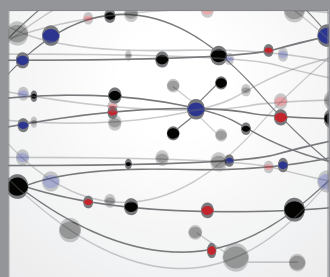

The Scientific World Journal
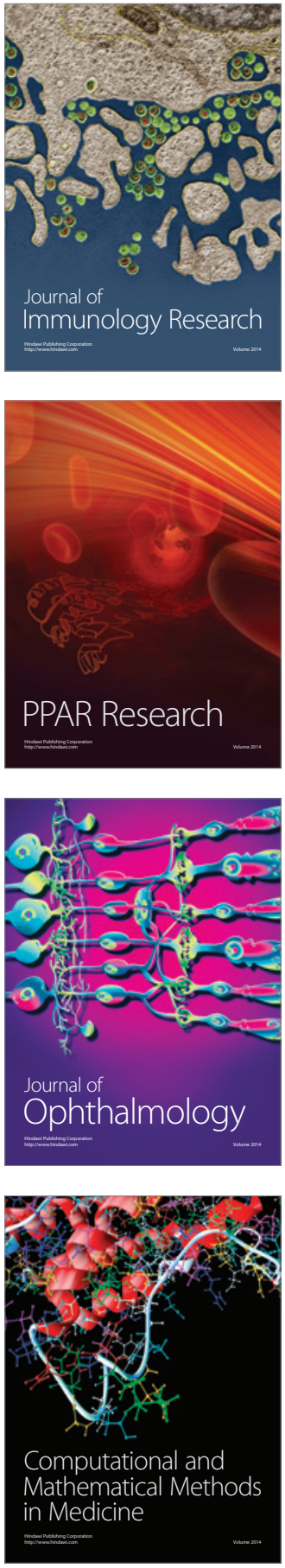

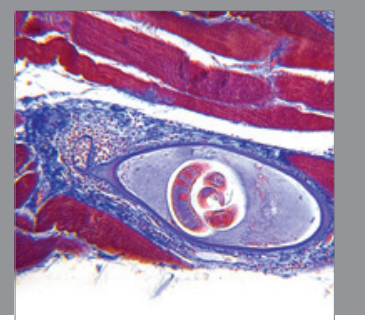

Gastroenterology

Research and Practice
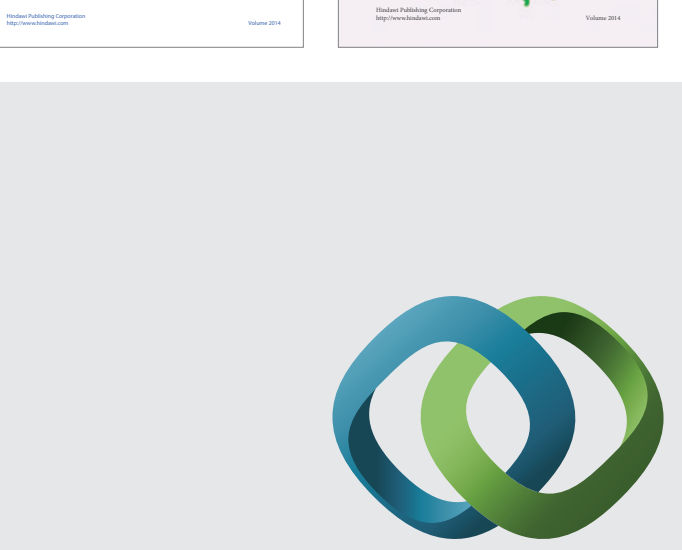

\section{Hindawi}

Submit your manuscripts at

http://www.hindawi.com
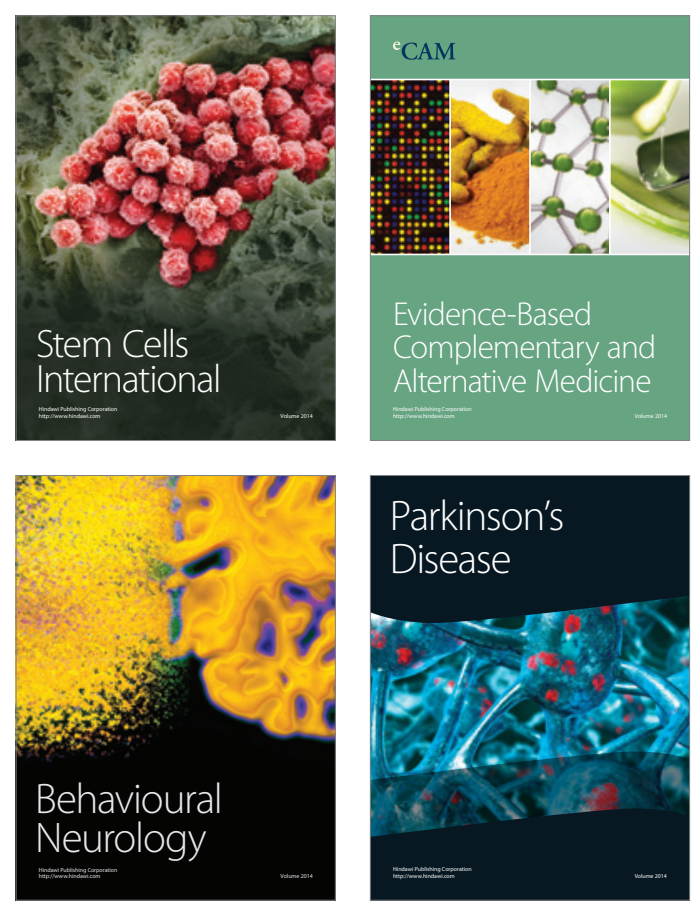

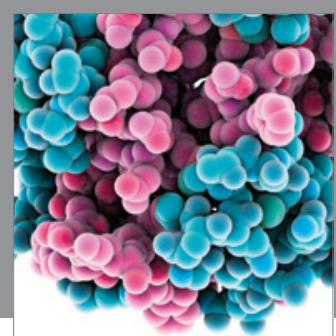

Journal of
Diabetes Research

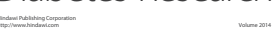

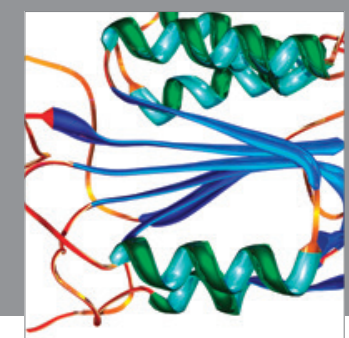

Disease Markers
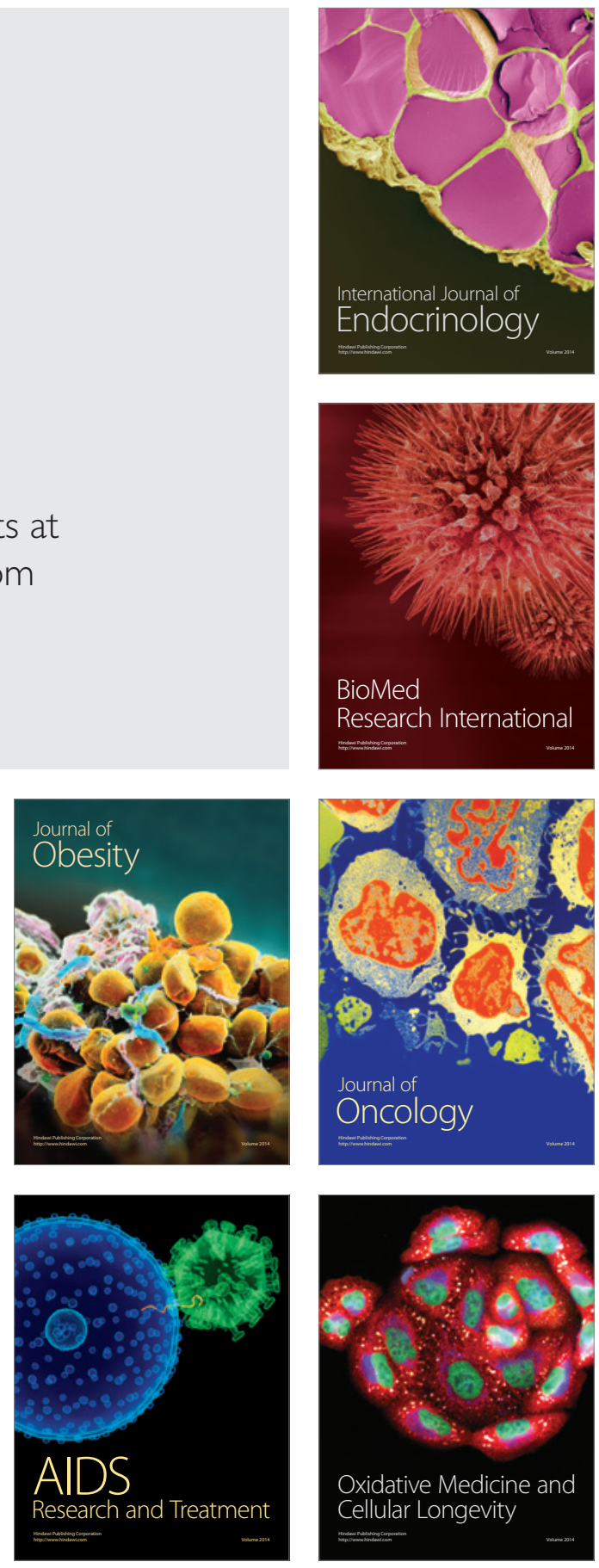